\title{
The Discussion of the further Studies of the Magnetic Suspension Vibration Isolator
}

\author{
Li Qiang ${ }^{1, a^{*}}$, Wei Shaoyan ${ }^{1, b}$, Liao Zhennan ${ }^{1, c}$, Jin Honglin ${ }^{1, d}$ \\ ${ }^{1}$ Wuchang University of Technology \\ alqyi1975@163.com, b1041697458@qq.com, '940919395@qq.com, d2194801778@qq.com
}

Keywords: Discussion, Magnetic suspension, Vibration isolator, further study

\begin{abstract}
The vibration isolators play an important role in the field of high resolution metrology and manufacturing, optical, physical and chemical experiments. Magnetic suspension vibration isolator (MSVI) is an efficient method to suppress the oscillations in vacuum environment. This paper firstly presents the requirements of the MSVI. Then, based on principle of the magnetic suspension, it introduces several types of the low frequency MSVI. Furthermore, the recent advanced investigations of the MSVI are reported such as magnetic force, eddy current damping effect, instability and the active control, etc. Finally, the author mainly discusses the future research contents of the MSVI and provides a new ideal for the development of the active MSVI. All of these will provide the research direction to develop the high performance of the MSVI.
\end{abstract}

\section{Introduction}

Vibration isolators are the key components in the fields of the micro-manufacturing, high-resolution metrology, optical, physical and experiments to constrain the external disturbances [1 3]. Some of them require vacuum compatibility, a contact- less structure, high force density and low stiffness [4]. For this application, the pneumatic vibration isolators with a low initial cut-off frequency are frequently applied to suppress the oscillations transmitted from the base to the equipment. But there are some limitations for the pneumatic vibration isolator to require a compressed air, which leads to a multitude of difficulty of application in vacuum environment [5]. However, with development of the permanent magnet material, samarium-cobalt or neodymium-iron-boron can gain the high residual magnetic induction, high coercive forces and hysteresis energy. Rare-earth permanent magnets are widely utilized in passive machine such as magnetic dampers, magnetic bearings, eddy brakes, levitation device and so on, especially in absence of the mechanical friction, the stiffness and damping properties can be easily modified by varying the air gap between the permanent magnets [4]. These magnetic suspension machines have famous features for the instance of being clean, noiseless and convenience to maintain [6]. Therefore, these properties of the permanent magnets appeal to more and more researchers and engineers to exploit a new kind of innovative magnetic levitation vibration isolator for the ultra-precision instruments and advanced manufacturing equipment.

Therefore, this paper introduces the advantages and the application prospects of the MSVI in section 1. Section 2 presents the theory of the magnetic suspension. Section 3 provides the overview of the basic configuration of the MSVI. Section 4 and Section 5 mainly discusses the further study content of the MSVI. Section6 closes this paper with some conclusions.

\section{The Theory of the Magnetic Suspension}

Applications of MSVIs have been discussed in clean circumstance with high precision positioning and high-speed transportation about decade years. The magnetic suspension systems can be classified into two types, ether repulsive force types or attractive one [7]. The former has an advantage that there is no energy required to float an object, which can be simplified into a magnetic spring-damping system. When the external oscillations act on the base of the MSVI, the 
amplitude of vibration can be attenuated, which are partly or even entirely consumed by the magnetic spring and realizes vibration isolation.

\section{The Basic Configuration of MSVI}

Based on the principle of the magnetic suspension, there are several kinds of MSVI to be presented, which are depicted in this section.

The Cylindrical MSVI. The basic configuration of the cylindrical MSVI is shown in Fig. 1 (a), which is composed of four couples of repulsive force type cylindrical permanent magnet [5]. When the load acts on the MSVI, the upper table and the load are floated by the lower one, the natural frequency of the MSVI is about $6.1 \mathrm{~Hz}$ in the vertical direction with the load capacity of $22 \mathrm{~kg}$ [5].

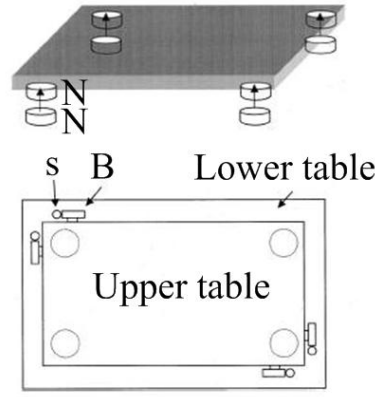

(a)

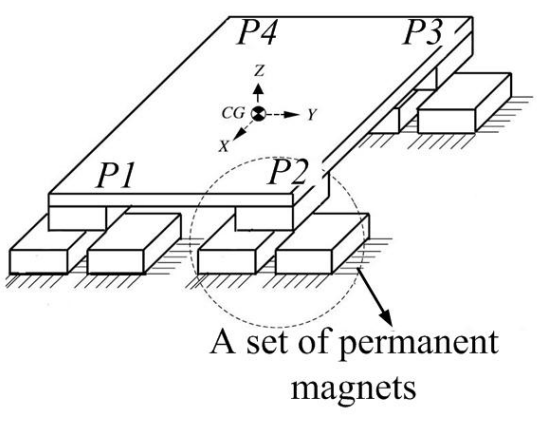

(b)

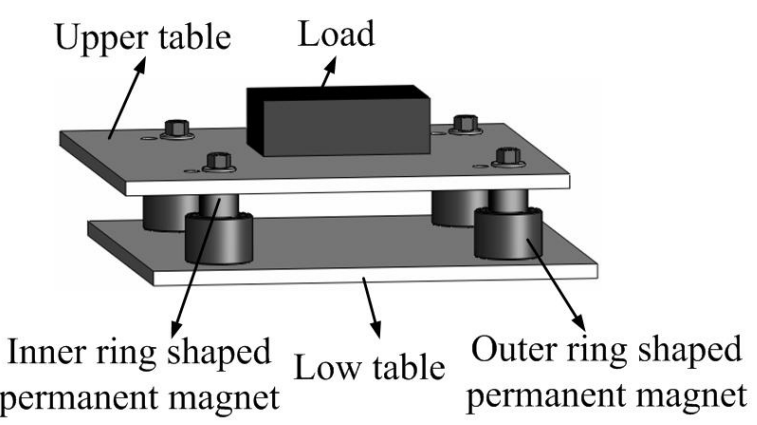

(c)

Fig. 1 The basic configuration of the MSVI.

(a) Cylindrical MSVI. (b) Cubical MSVI. (C) Ring-shaped MSVI [3, 5, 7].

The Cubical MSVI. Choi [7] proposed a kind of cubical MSVI in Fig. 1 (b), which consists of four couples of cubical permanent magnets. The two lower permanent magnets are attached to the fixed frame and an upper permanent magnet is movable. The MSVI can realize vibration isolation of 5 DOF due to the instability of the repulsive force type permanent magnet. In order to overcome the instability, there is an orientation that it requires to control. The natural frequency of the MSVI ranges from to $2 \mathrm{~Hz}$ to $5.88 \mathrm{~Hz}$. Choi [7] also predicted the MSVI can be applied in a clean environment or a vacuum one.

The Ring-Shaped MSVI. Another MSVI consists of four couples of ring-shaped permanent magnets in Fig. 1(c). The inner magnetic ring is magnetized axially and the outer one is magnetized radially. The upper table and the load are levitated by the lower permanent. The natural frequency of this MSVI is about $6 \mathrm{~Hz}$ with the load of $23.6 \mathrm{~kg}$ [3].

Till now, there are only three kinds of MSVI reported in the bibliography $[3,5,7]$. Although some properties of the MSVIs is superior to the other vibration isolator, the researchers and engineers can't acquaint the behavior of magnets entirely, which still requires a further studies on the MSVI adequately.

\section{The Further Studies of the MSVI}

In order to extend to research on the MSVIs, this section presents the recent studies of the MSVI such as magnetic force and stiffness, damp, instability and active control as follows.

The Magnetic Force and Stiffness. In order to enhance the vibration isolation performance of the MSVI, it is important for the MSVI to calculate the magnetic force and stiffness. Although there are several ways of obtaining the magnetic forces between the magnets, the authors generally use numerical means or 2-D analytical calculations to determine magnetic forces [8]. The disadvantage of a numerical approach is that it needs a high computational cost and the numerical algorithms. It is difficult to acquire the general properties of the permanent magnets. Consequently, the authors try to find the semi analytical expressions which are very essential steps to evaluate magnetic force [9, 10]. The major problem of a semi analytical expression only can calculate the magnetic force of the 
fixed dimension of the magnets. It is difficult to use the semi model to optimization design and analyze the properties of the magnetic force. Furthermore, the analytical model is really required. Vokoum [11] provided a model of attraction force between the arrays of cylindrical permanent magnets. Zhu [3], based on the 2-D analytical model, established the magnetic force analytical expression for a kind of the ring-shaped permanent magnet. Akoun [12] provides an analytical magnetic force model between two cubical magnets. They have been applied in the design of the MSVI. However, they can only gain few kinds of analytical models of the magnetic force for the permanent magnets.

Eddy Current Damping Effect. Eddy currents are generated in a conductor in a time-varying magnetic field due to Faraday's law. They are induced in the MSVI either by the movement of the conductor fixed or connected with the permanent magnet or by changing the strength of the magnetic field, initial motional and transfer electromotive forces (EMSF) [13]. When the oscillations act on the MSVI, the permanent magnets generate a time-varying magnetic field in both horizontal and vertical directions. They can generate the EMSF, which are based on a time-varying magnetic field and the relative motion of the conductor of the MSVI, respectively. The vibration energy of the loads is converted into heat via eddy current induction and dissipated through the conductor part [14]. However, motional and transformer effects are relative with the position, size dimension, material air gap distance, velocity, frequency and amplitude of the oscillation of the conductor and magnets and so on. Only few bibliographies [13-15] gave the damping characteristic of the eddy currents, which can be as a further study for the MSVI.

The Instability of the Permanent Magnets. Earnshaw [16] proposed and proved that a passive magnetic suspension device is unstable at least one direction. The conferences have reported that there are two methods to stabilize the passive MSVI at equilibrium position. One is mechanical constraint. Puppin [3] proposed a MSVI. The upper table of the MSVI is mechanically confined in the equilibrium position with four ball bearings connected to the table and sliding along four vertical shafts. Zhu [3] applied the air bearing, which constrains the motional direction of the MSVI. It only can isolate the oscillation in vertical orientation. Bonisoli [4] designed a DOF passive MSVI with the mechanical spring in parallel with cubical permanent magnets and corrected the horizontal instability with vertical guides. The other method is active control. Choi [7] applied the linear motor to overcome the instability of the passive MSVI. All of these methods can influence on performance of the vibration isolation of the MSVI, even negative effects.

The Active MSVI. Subrahmanyman [19] has demonstrated that the passive vibration isolators attenuates high frequency disturbances with reasonable attenuation rate while the active system mainly constrains the low frequency vibration. For the purpose of enhancing low frequency vibration isolation performance of the present MSVI, it needs to explore the active MSVI with actuators, sensors and controller, which is an efficient method to enhance the low frequency of the MSVI. Banik [18] implemented the voice motor in an MSVI which works to control the vibration typically in range from 0.1 to $100 \mathrm{~Hz}$ in all six degrees of freedom. Roberson [19] designed a non-linear controller for zero stiffness magnetic spring. The simulation results indicate that active control can improve the vibration isolation of the passive MSVI. Another few bibliographies [20-22] also have reported that the active MSVIs have been researching on. However, there are many difficulties to obtain the excellent vibration isolation performance practically.

\section{Discussion}

Above all, although the present works partly focus on the properties of the MSVI, which have been presented in the former section, it obtains the excellent vibration isolation performance. There still requires discussing the further studies of the MSVI.

First of all, only three kinds of permanent magnet applied in development of MSVI, such as Cylindrical, Cubical and Ring-shaped permanent magnet. The natural frequency of the MSVI is $5 \mathrm{~Hz}$ or so. Compared with the current passive air spring, it needs to propose the optimal structure or novel configurations to enhance the vibration isolation performance of the MSVI with a minimal 
stiffness and maximal load capacity, which demand the precise analytical magnetic force and stiffness model.

However, there are only few bibliographies provided the analytical magnetic force and stiffness model for the permanent magnet, which has been already applied to exploit the MSVI [7, 12, 19]. There haven't the analytical models of the other basic configurations. Up to now, there are three kinds of methods to build up the magnetic model such as Colombian approach, fundamental laws of magetostatics (Biot Savart Law, Lorentz Force, and Maxwell's Equations) and interaction energy [23]. The former two methods usually can establish the semi analytical expressions [9, 24, 25]. The latter may set up the analytical expression [3]. All of these can't meet the requirement of exploring the innovative MSVI.

Moreover, there exists a challenge to deal with the instability of the MSVI. The present bibliography provided few approaches to overcome the instability of the permanent magnet such as mechanical devices and the active control $[3,5,7]$. The former can influence the vibration isolation performance of the MSVI due to the mechanical friction. The latter has a complicated structure and expensive cost. The controlled direction can't obtain vibration isolation. It needs to find a novel method to solve the instability of the MSVI.

Furthermore, with the rapid development of the precision measuring instruments, the micro-or nano-scale measurements or processes are very sensitive to ground vibrations. Although the passive pneumatic vibration isolator can be designed to achieve low natural frequency in the range of $2-4 \mathrm{~Hz}$, the higher performance vibration isolators are still needed to meet the requirements of the corresponding vibration tolerance. The PID and fuzzy control has been applied in the active pneumatic vibration isolator [26-28]. It obtained the relative low cut-off frequency and wide bandwidth.

Generally, a six degree of freedoms (DOFs) vibration isolator is composed of four vibration isolation unit, as shown in Fig. 2. It may be broken down into the vibration isolation of the two horizontal directions(x and y direction) and a vertical orientation. Namely, the vibration isolation of the two horizontal directions combined with a vertical orientation can realize the ones of six DOF. $\mathrm{Khx}$ and Khy represent the stiffness of an isolator in horizontal orientation and $\mathrm{Kv}$ is the stiffness of the isolator in vertical direction in Fig. 2, respectively.

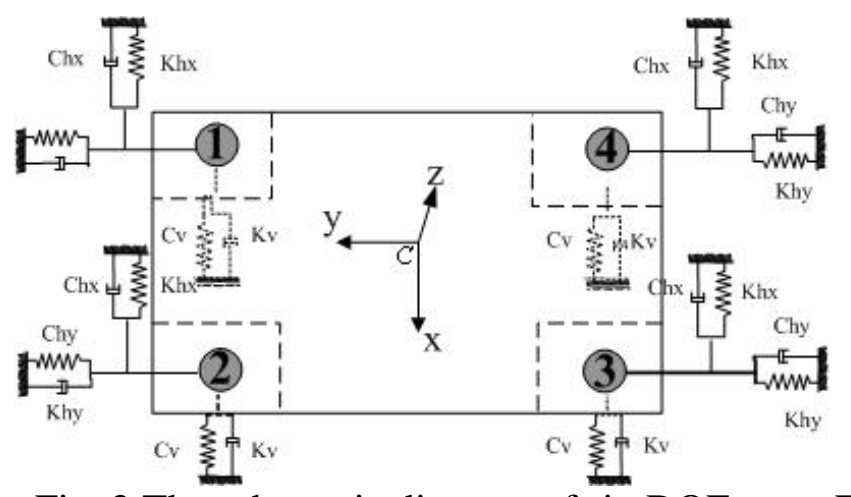

Fig. 2 The schematic diagram of six DOF vibration isolation.

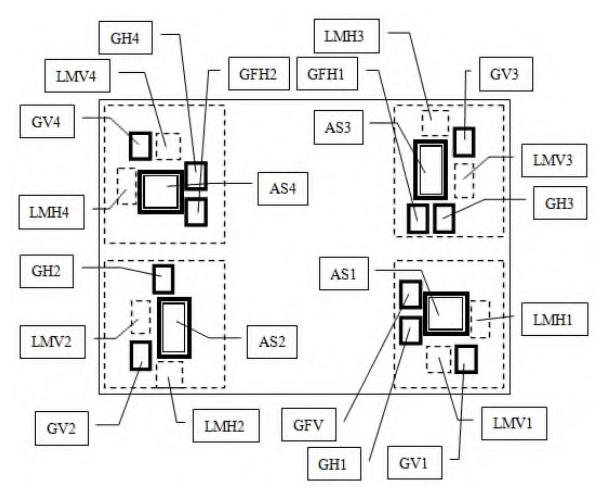

Fig. 3 The schematic diagram of six DOF active vibration isolation [29].

As a case study, Fig. 3 represents the schematic diagram of six DOF active dual-chamber pneumatic vibration isolators, which is composed of four air spring vibration isolation unit, four voice coil motor, and eleven velocity sensor. The symbols in Fig. 4 describe in Table 1. The PID control is applied in the active dual-chamber pneumatic vibration isolator [29]. 
Table 1. The component of the active dual-chamber pneumatic vibration isolator.

\begin{tabular}{|l|l|}
\hline Symbols & Description \\
\hline AS1,AS2,AS3,AS4 & Vibration isolator unit \\
\hline LMV1,LMV2,LMV3,LMV4 & The voice coil in vertical direction. \\
\hline LMH1, LMH2, LMH3, LMH4, & The voice coil in horizontal direction. \\
\hline GV1,GV2,GV3,GV4 & The sensor in vertical direction \\
\hline GH1,GH2,GH3,GH4 & The sensor in horizontal direction \\
\hline GFV & The sensor in vertical direction on the base \\
\hline GFH1,GFH2 & The sensor in horizontal direction on the base \\
\hline
\end{tabular}

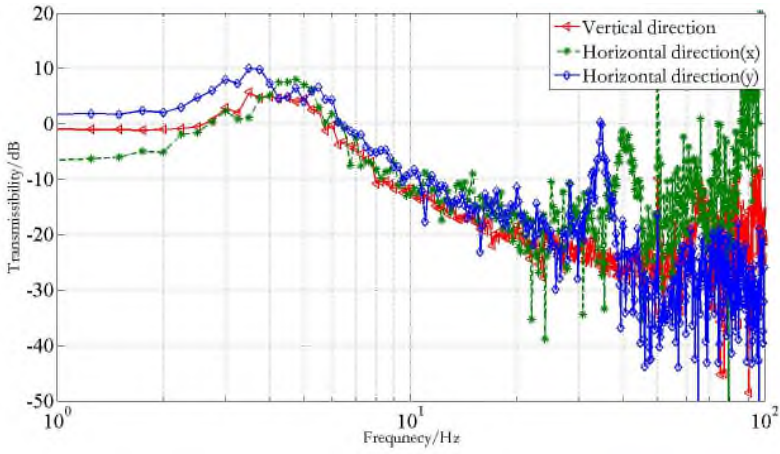

(a)

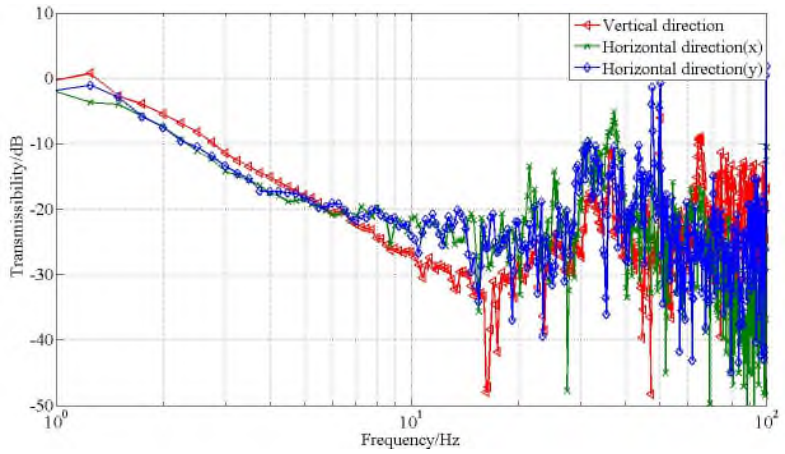

(b)

Fig. 4 The transmissibility of the dual-chamber pneumatic vibration isolator.

(a) Passive vibration isolator. (b) Active vibration isolator [29].

Compared with testing data between the passive and active dual-chamber pneumatic vibration isolator, the experimental results demonstrate the active dual-chamber pneumatic vibration isolators can obtain low-frequency vibration isolation. It demonstrates that the performance of active vibration isolator is superior to that of the passive one.

As another case study, Kerber [30] adopted the linear spring to exploit an active vibration isolator. Velocity feedback, PI or $\mathrm{H} \propto$ controller is applied in the active linear spring, respectively. The simulation results indicate that they all can strengthen the performance of the linear passive vibration isolator. The $\mathrm{H} \propto$ controller is a more effect method than the others. All of these demonstrate that active control is an effective method to improve the performance of the vibration isolator. Therefore, these control-methods for the air and linear springs provide effective ideals to develop novel active MSVI, Which may be used to research on the active MSVI. It will be the further studies to the author.

\section{Conclusions}

This paper mainly discusses the basic configuration, analytical magnetic force and stiffness model, magnetic damping, instability and active control of the present MSVI and so on. The aim is to seek the new research contents for the MSVI. It improves the vibration isolation performance of the MSVI quickly and extends the MSVI applying in the fields of high resolution metrology and manufacturing, optical, physical and chemical experiments widely.

\section{References}

[1] J. L. G. Janssen, E. A. Lomonova, Analytical Force, Stiffness, and Resonce Frequnecy Calculations of a Magnetic Vibration Isolator for a Microbalance, IEEE Trans. Ind. Appl. 51(1) (2015) 204-210. 
[2] B. Li, W. Zhao, Z. Deng, Modeling and analysis of the a multi-dimensional vibration isolator based on the parallel mechanism, J. Manuf. Syst. 31(1) (2102) 50-58.

[3] Y. Zhu, Q. Li, D. Xu, M. Zhang, Modeling of Axial Force and Stiffness of Ring-Shaped Permanent-Magnet Passive Vibration Isolator and its Vibration Isolating Experiments, IEEE Trans. Magn. 48(7) (2012) 2228-2238.

[4] E. Bonisoli, A. Vigliani. Passive effects of rare-earth permanent magnets of flexible conductive structures, Mech. Res. Commun. 33(3) (2006) 302-319.

[5] E. Puppin, V. Fratello, Vibration isolation with magnetic springs, Rev. Sci. Instr. 73(11) (2002) 4034-4036.

[6] J. M. D. Coey, Permanent magnet applications, J. Magn. Magn. Mater. 248(3) (2002) 441-456.

[7] K. B. Choi, Y. G. T. Cho, S. Shin, et al. Stabilization of one degree-of-freedom control type levitation table with permanent magnet repulsive forces, Mechatron. 13(6) (2003) 587-603.

[8] J. Bart. J. Gysen, J. Messen. 3D analytical and numerical modeling of skewed tubular magnet arrays, Electromagnetic Field Computation, Chicago, USA, 2010.

[9] R. Ravaud, G. Lemarquand, V. Lemarquand, Force and stiffness of passive magnetic bearings using permanent magnets: part1: axial magnetization, IEEE Trans. Magn. 45(7) (2009) 2996-3002.

[10] S. A. J. Hol, E. Lomonova, A. J. A. Vandenput. Design of a magnetic gravity compensation system, Prec. Eng. 30(3) (2006) 265-273.

[11] D. Vokoun, G. Tomassetti, M. Beleggia et al. Magnetic force between arrays of cylindrical permanent magnets, J. Magn. Magn. Mater. 323(1) (2011) 55-66.

[12] G. Akoun, J. P. Yonnet, 3D analytical calculation of the forces exerted from two cubical magnets, IEEE Trans. Magn. 20(5) (1984) 1962-1964.

[13] C. Elbuken, E. Shameli, M. B. Khamesee. Modeling and analysis of eddy-current damping for high-precision magnetic levitation of a small magnet, IEEE Trans. Magn. 43(1) (2007) 26-32.

[14] B. Ebrahimi, M. B. Khamesee, M. F. Golnaraghi, Design and modeling of a magnetic shock absorber based on eddy current damping effect, J. Sound Vib. 315(4) (2008) 875-889.

[15] B. Ebrahimi, M. B. Khamesee, M. F. Golnaraghi. Eddy current damper feasibility in automobile suspension: modeling, simulation and testing, Smart Mater. Struct. 18(1) (2009) 1-12.

[16] S. Earnshaw. On the nature of the molecular forces which regulate the constitution of the luminiferous ether, Trans. Combridge Philos, 7(1) (1839) 97-112.

[17] P. K. Subrahmanyman, A modal approach to precision motion control, Ph. D. dissertation, Massachusetts Institute of Technology, The USA: 1999.

[18] R. Banik, D. G. Gweon. Design and optimization of voice motor for application in active vibration, Sens. Actuat. A, 137(2) (2007) 236-243.

[19] W. Roberson, R. Wood, B. Cazzolato, et al. Zero-stiffness magnetic springs for active vibration isolation, Proceeding of the 6th International Symposium on Active Noise and Vibration Control, Adelaide, Australia, 2006, pp. 589-597.

[20] J. Fu, P. Li G. Liao, M. Yu, Development and Dynamic Characterization of a Mixed Magnetorheological Elastomer Isolator, IEEE Trans. Magn. DOI10. 1109/TMAG. 2016. 2606406, 2016. 
[21] S. Ito, D. Neyer, S. Piker et al, Atomic Force Microscopy Using Voice Coil Actuators for Vibration Isolation, 2015 IEEE International Conference on Advanced Intelligent Mochatronics, Busan, Korea, 2015, pp, 470-475.

[22] P. Li, J. Fu, Y. Wang et al, Dynamic Model and Parameters Identification of Piezoelectric Stack Actuators, 2014 26th Chinese Control and Decision Conference, Changsha, China, 2014.

[23] R. Ravaud, G. Lemarquand, S. Babic et al. Cylindrical magnets and coils: fields, forces, and inductances, IEEE Trans. Magn. 46(9) (2010) 3585-3590.

[24] S. A. J. Hol, E. A. Lomonova, A. J. A. Vandenput, Design of a magnetic gravity compensation system, Prec. Eng. 30(3) (2006) 265-273.

[25] R. Ravaud, G. Lemarquand, V. Lemarquand, Force and stiffness of passive magnetic bearings using permanent magnets: part2: radial magnetization, IEEE Trans. Magn. 45(9) (2009) 3324-3342.

[26] Y. Gai, Y. Liu, Q. Guo, et al. Study on active vibration isolation system for the bed of ultra-precision turning machine, International Technology and Innovation Conference, Weihai, China, 2006, pp. 960-964.

[27] Ch. Hua, Research on the vibration isolation characteristic of dual-chamber pneumatic spring, Ph. D. dissertation, Beijing, Tsinghua University, 2011.

[28] H. Yan, Modeling and active control ultra-precision vibration isolation system, Ph. D. dissertation, Wuhan, Huazhong University of Science and Technology, 2012.

[29] J. Huang, Control research of a table top active vibration isolator based on the PID algorithm, M. S. dissertation, Chengdu, University of Electronic Science and Technology of China, 2014.

[30] F. Kerber, S. Hurlebaus, B. M. Stoener, U. Control concepts for an active vibration isolation system, Mech. Syst. Sign. Proc. 21(8) (2007) 3042-3059. 